\title{
Jaroslav J. Polivka y el Guggenheim Museum de New York
}

\author{
Jaroslav J. Polivka and the Guggenheim \\ Museum of New York
}

D. Martín-Sáiz ${ }^{*)}$

\section{RESUMEN}

El Guggenheim Museum es un claro ejemplo de arquitectura influenciada por su estructura. La arquitectura de Frank Lloyd Wright se nutre de una fusión especial con la estructura, en ocasiones difícil de diferenciar de otros elementos. Esto llevó a Wright hacia planteamientos novedosos, como el de las láminas de hormigón armado, que implicaban un mayor grado de complejidad técnica.

Jaroslav Joseph Polivka aportó tanto sus conocimientos específicos sobre el material y sus métodos de análisis como una especial sensibilidad hacia el papel formal de la estructura dentro de la arquitectura. En este sentido, la lámina en espiral del edificio del museo sobrepasaba los límites razonables del momento de esta tipología, por lo que Polivka tuvo que complementar la base teórica disponible con ensayos que corroborasen el método propuesto, todo ello enfocado hacia la materialización del espacio continuo ideado por Wright (1).

142-165

Palabras clave: Arquitectura, estructura; Frank Lloyd Wright; Jaroslav Joseph Polivka; Guggenheim Museum; Eduardo Torroja; arquitectura orgánica; placas y láminas.

\section{SUMMARY}

The Guggenheim Museum is a clear example of architecture influenced by its structure. The architecture of Frank Lloyd Wright feeds on a special merger with the structure, sometimes difficult to differentiate from other elements. This led Wright to new approaches, such as reinforced concrete plates, implying a greater degree of technical complexity.

Jaroslav Joseph Polivka provided both specific knowledge about the material and methods of analysis, as a special sensitivity regarding the formal role of structure in architecture. In this sense, the spiral ramp of the museum exceeded the reasonable limits of this type in his time and Polivka had to supplement the available theoretical with essays to substantiate the proposed method, all this focused on the materialization of a continuous space devised by Wright (1).

Keywords: Architecture; structure; Frank Lloyd Wright; Jaroslav Joseph Polivka; Guggenheim Museum; Eduardo Torroja; organic architecture; plates and shells.

(*) Dr. Arquitecto. Delegado de BOMAINPASA. 


\section{COLABORACIONES ENTRE ARQUITECTOS E INGENIEROS}

Durante la evolución de la arquitectura del siglo XX, así como en muchos casos la estructura fue separándose del resto de la arquitectura, el trabajo realizado por los diferentes profesionales que intervenían en los proyectos arquitectónicos también fue disociándose. Ha sido habitual que algunos arquitectos desarrollen un proyecto sin haber pensado en la solución estructural, contando con que, a posteriori, un ingeniero resolverá la estructura de la forma menos molesta posible. De este planteamiento nunca se ha obtenido un buen resultado ya que, al separar tan drásticamente arquitectura y estructura, se eliminan otras funciones arquitectónicas de la estructura.

En cambio, esta separación "forzada" es en otros casos inexistente (2). Los grandes arquitectos han sabido mantener la unidad entre arquitectura y estructura y, habitualmente, esto ha sido posible gracias al trabajo conjunto con un consultor de estructuras, no sólo resolviendo el molesto problema de la gravedad, sino como colaborador y portador de soluciones arquitectónicas (3). Incluso hay numerosos ejemplos donde los arquitectos han desarrollado un trabajo técnicamente sofisticado y los ingenieros han asumido un papel de tipo arquitectónico, invirtiéndose así unas tareas predeterminadas a priori. Así se funden arquitectura y estructura en un solo concepto, desdibujando los límites de cada una de ellas (4).

Desde Joseph Paxton y Charles Fox en el Crystal Palace de Londres hasta Arata Isozaki y Mamoru Kawaguchi en el Centro de Convenciones de Nara, por ejemplo, se puede hacer un listado muy extenso de colaboraciones fructíferas en este sentido (5) (6). Un ejemplo cercano es el de Carlos Arniches y Martín Domínguez con Eduardo Torroja en el Hipódromo de la Zarzuela. Mies van der Rohe también colaboraba habitualmente con el ingeniero de estructuras Frank J. Kornacker, que participó en los proyectos del Convention Hall o de los apartamentos Lake Shore Drive entre otros. O el trabajo de Louis Kahn y August Komendant, que está ampliamente documentado en el libro 18 años con el arquitecto Louis I. Kahn (7). Un poco más avanzado el siglo XX, Kenzo Tange y Oscar Niemeyer contaron de forma continuada con las contribuciones de ingenieros de estructuras. Yoshikatsu Tsuboi trabajó con Tange en los proyectos de mayor envergadura, como la catedral de Tokio o los gimnasios de Yoyogi. Y Joaquim Cardozo, colaborador habitual de Niemeyer, le permitió explorar las posibilidades plásticas del hormigón; como discípulo de Le Corbusier que era, pensaba en una concepción simultánea de arquitectura y estructura (8) (9) (10).

En definitiva, los maestros de la arquitectura del siglo XX han sido plenamente conscientes de la importancia formal de la estructura dentro de la arquitectura (11) (12) (13). En este sentido, Frank Lloyd Wright es también una notoria muestra de ello. Hay que tener en cuenta que su formación inicial fue en ingeniería y no en arquitectura, involucrándose en la resolución de los problemas técnicos ya desde su juventud. Wright entró a trabajar en la oficina del ingeniero Allan Conover, desde donde pudo acceder a la Universidad de Wisconsin y de allí al estudio de Adler y Sullivan. Una vez independizado, durante el periodo de las casas de la pradera, las soluciones ingenieriles de Wright se solían basar más en la intuición que en exhaustivos cálculos. Pero a partir del primer edificio de mayor escala, el Larkin de 1903, Wright requirió asesoramiento, por lo que recurrió a un ingeniero de reconocido prestigio en Chicago, Paul Mueller, habitual de Adler y Sullivan y especialista en estructuras de acero, que acabó colaborando en la ejecución de los tres primeros edificios mayores de Wright (Unity Temple, 1906; Midway Gardens, 1912, y el Hotel Imperial de Tokio, 1916-22) (14). Hasta entonces Wright había confiado en su aproximación intuitiva a las estructuras, con resultados decididamente variados, pero a raíz de la construcción de estos edificios de mayor envergadura parece que asumió la necesidad de colaborar con ingenieros de estructuras de forma continua. En 1935 Mendel Glickman y William Wesley Peters entraron a formar parte del equipo habitual de Taliesin, hasta que, finalmente, Polivka, se incorporó también como consultor externo a partir del proyecto del Guggenheim Museum. Polivka intentó reivindicar durante los últimos años de su vida un reconocimiento sobre sus aportaciones realizadas a la arquitectura de Wright. Bien fuera por la fuerte personalidad del arquitecto o por la inercia de la sociedad a converger la arquitectura en una sola persona, Polivka, como muchos otros, no tuvo un prestigio proporcionado a sus aportaciones. Huelga decirlo, con este trabajo se desea otorgarle un mérito justo por su trabajo desarrollado junto a Wright. 


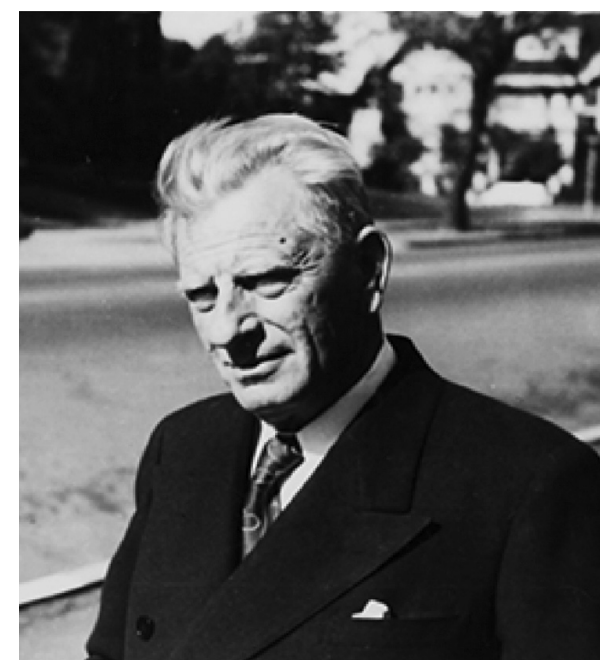

\section{LA FIGURA DE JAROSLAV JOSEPH POLIVKA}

El Dr. Polivka (Figura 1) nació en Praga en 1886 y se licenció en la Escuela de Tecnología de la misma ciudad en 1909, donde también se doctoró en 1917. Después de la Primera Guerra Mundial, se estableció como arquitecto e ingeniero y empezó a desarrollar sus habilidades en el análisis de tensiones, llegando a ser un experto en el estudio tensional mediante modelos foto-elásticos. Polivka ganó reconocimiento internacional por su diseño del Pabellón Checo en la Exposición de Paris de 1937 en colaboración con el arquitecto Jaromír Krejcar. También trabajó con Kamil Roscot en el diseño de otro pabellón, en este caso para la New York World's Fair. En 1939 emigró a Estados Unidos y tomó posición como investigador asociado y conferenciante de la Universidad de California, Berkeley, donde lideró el laboratorio de fotoelasticidad junto a Victor di Surevo (15). Este sistema de análisis permitía estudiar de forma visual el comportamiento laminar de un material, siendo un método precursor del método de elementos finitos. Su aplicación podía resultar de gran ayuda de cara a complementar un análisis estrictamente numérico y Polivka así lo plasmó en un texto titulado Analysis of Gravity Load Stresses by Photoelastic Methods ${ }^{1}$, como método alternativo para el análisis de láminas sometidas a tensión plana.

Polivka conocía a Wright a través de las publicaciones europeas de sus obras, anteriores a 1930, que tuvieron gran transcendencia a la hora de difundir en Europa su obra temprana. La admiración por los proyectos de Wright se contextualizó en el ambiente universitario donde desarrollaba las investigaciones, e incluso su compañero de universidad

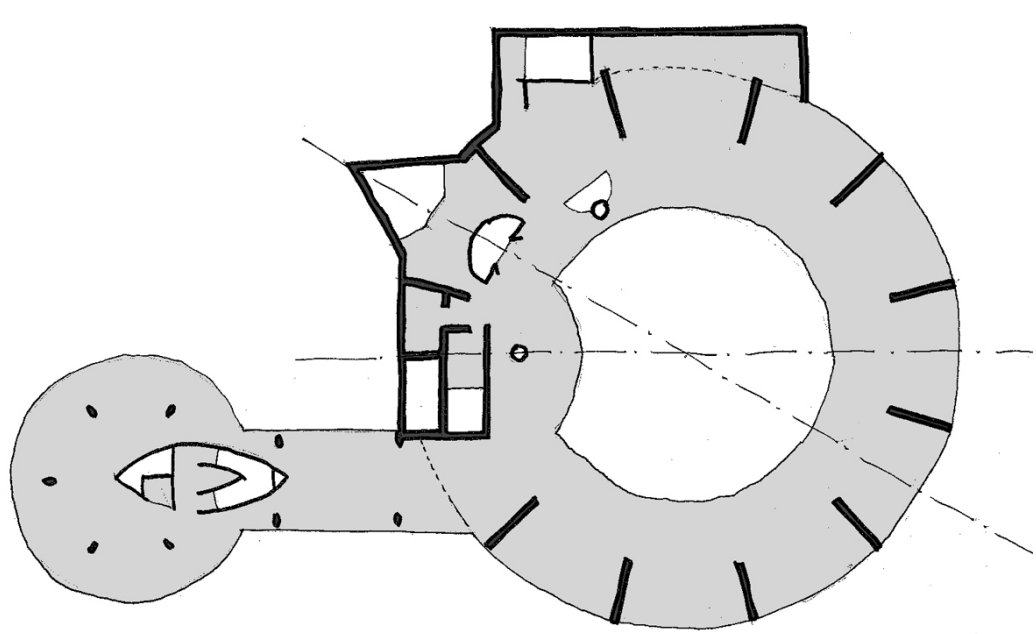

2

Antolin Raymond, arquitecto checo que también emigró a Estados Unidos, trabajó para Wright en la redacción del proyecto del Hotel Imperial en Tokio (16).

Cuando Polivka llegó a Estados Unidos y retomó el contacto con antiguos compañeros europeos, recuperó también el entusiasmo por poder llegar a conocer a Wright personalmente. En enero de 1946 Wright había publicado en la revista Architectural Forum (17) un artículo referente al proyecto de la Modern Gallery, donde Wright criticaba de forma directa a los ingenieros, incapaces de resolver el problema de la espiral del museo sin la incorporación de pilares en el borde interior de la rampa. A raíz de esto, Polivka se animó a contestarle mediante una carta, exponiendo sus ideas respecto de los modelos estructurales basados en la naturaleza. En ese momento Polivka era ya una autoridad en diseño estructural, aunque lo más destacable era su intuición e innovación en el análisis de modelos foto-elásticos. Sin embargo, la colaboración no se inició por destacar ambos en sus respectivas disciplinas, sino debido a la frustración de Wright frente a las dificultades de materialización de la rampa del museo, problema para el cual Polivka sugería una solución. A partir de un primer intercambio de impresiones, Polivka se desplazó a Taliesin y comenzó a trabajar en el desarrollo de la estructura de la Modern Gallery. Debido a la dilatación en el tiempo del desarrollo del proyecto, tuvieron oportunidad de compartir otros seis trabajos. Ordenados cronológicamente, son (15):

1. The Modern Gallery (The Guggenheim Museum) New York, 1943-1959 (Figuras 2 y 3). Wright propuso para el arte no convencional un edificio también poco convencional, desarrollado a través de una rampa en forma de espiral.
${ }^{1}$ Polivka, Jaroslav J. Illinois Institute of Technology 1942. Texto citado en "Philosophy of structures", Eduardo Torroja.

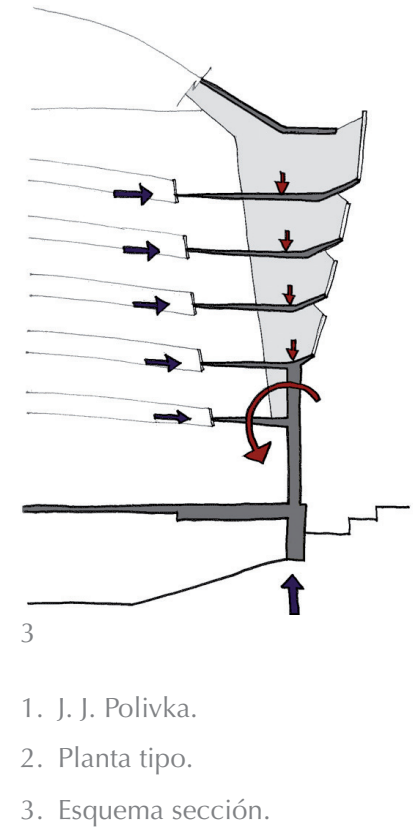




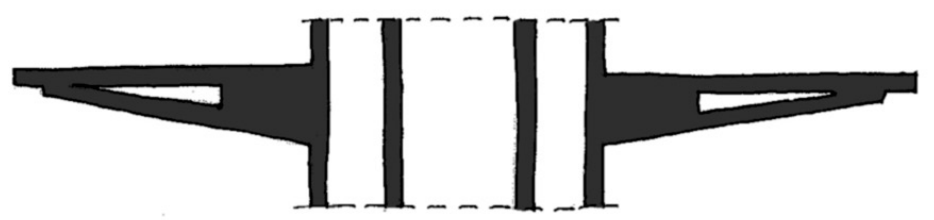

4

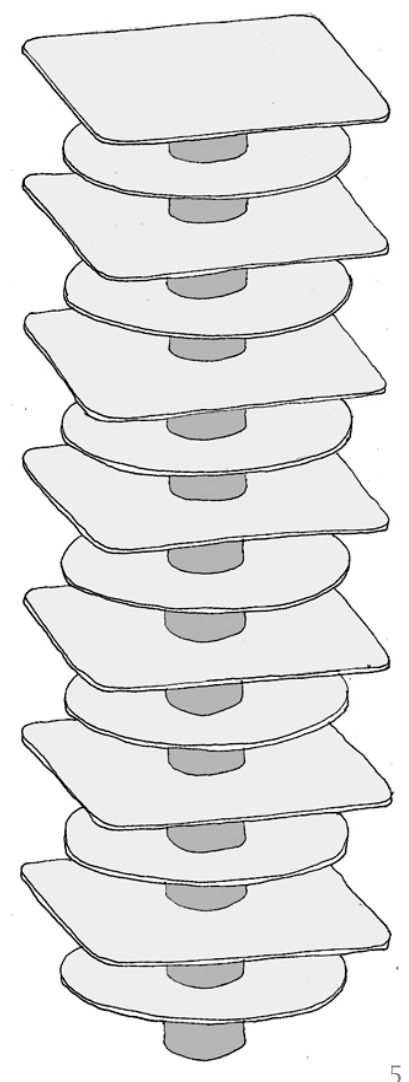

Polivka le proporcionó sus conocimientos sobre comportamiento laminar y el análisis estructural mediante modelos foto-elásticos, para justificar ante las autoridades de New York que el edificio era seguro. El trabajo de Polivka permitió a Wright eliminar soportes en el interior de la rampa siendo así la estructura coherente con sus planteamientos arquitectónicos.

2. The Johnson Wax Tower, Racine, Wisconsin, 1946. En 1936 Wright había proyectado el edificio administrativo de la misma compañía con su famosa retícula de columnas dendriformes. Para la ampliación diseñó una torre de laboratorios en la que, desde un potente soporte central, los forjados surgían en voladizo (Figuras 4 y 5) "como las ramas de un árbol". Polivka se encargó del análisis y diseño de la estructura, incluyendo para su cimentación el original sistema de raíz pivotante, tap-root (Figura 6), utilizado también en otros proyectos.

3. The Rogers Lacy Hotel, Dallas Texas, 1946. Con sus cincuenta y cinco plantas, pretendía ser el edificio más alto al oeste del Mississippi. Wright proponía cerrarlo con espectaculares paneles de vidrio en forma de diamante, para lo cual Polivka también le asesoró gracias a sus conocimientos sobre las propiedades de este material. Los forjados se proyectan en voladizo en los dos sentidos a partir de los núcleos de comunicaciones (Figura 7).

4. The V.C. Morris House, San Francisco, California, 1949. Vivienda al borde de un acantilado desarrollada a partir de círculos concéntricos de hormigón armado, algunos en voladizo (Figura 8). Polivka investigó la posibilidad de utilizar unos paneles aislantes gunitados, para aligerar la estructura y mejorar el comportamiento térmico del edificio.

5. The Butterfly Bridge, San Francisco, California 1949. El arco de hormigón armado para cruzar la bahía de San Francisco, idea de Polivka, resultó ser el elemento más significativo del proyecto de Wright. Posteriormente los "arcos de mariposa" (Figura 9) fueron utilizados por Wright como recurso formal en muchos otros proyectos. La propuesta ofrecía considerables ventajas económicas y de mantenimiento respecto de una solución convencional de puente en celosía de acero. El proyecto del Butterfly Bridge representó la perfecta simbiosis entre arquitectura e ingeniería, siendo el cénit de su colaboración.

6. The Belmont Racetrack Pavilion, Long Island, New York, 1956. Estructura de cables de acero con forma de catenaria de la que se suspendía una enorme cubierta de paneles plásticos. Polivka estudió y analizó detalles, materiales y costes para los aproximadamente $41.800 \mathrm{~m}^{2}$ de la estructura (Figura 10).

7. The Mile High Illinois Building, Chicago, Illinois, 1956. El proyecto del rascacielos incorporaba varios de los elementos propuestos en el Rogers Lacy Hotel y en la Johnson Tower Research, pero a la escala de $1.609 \mathrm{~m}$ de altura. En este proyecto Polivka analizó las oscilaciones de otras estructuras históricas en altura. A partir de sus resultados, investigó la manera de eliminar las vibraciones en esta torre que hicieran posible su construcción (Figura 11).

De esta relación únicamente los dos primeros proyectos llegaron a construirse, pero su análisis demuestra la importancia del papel que Polivka jugó en estas obras, no sólo como ingeniero estructural, cuya experiencia es innegable, sino también por su sensibilidad arquitectónica a la hora de entender los límites formales y potencial del hormigón armado.
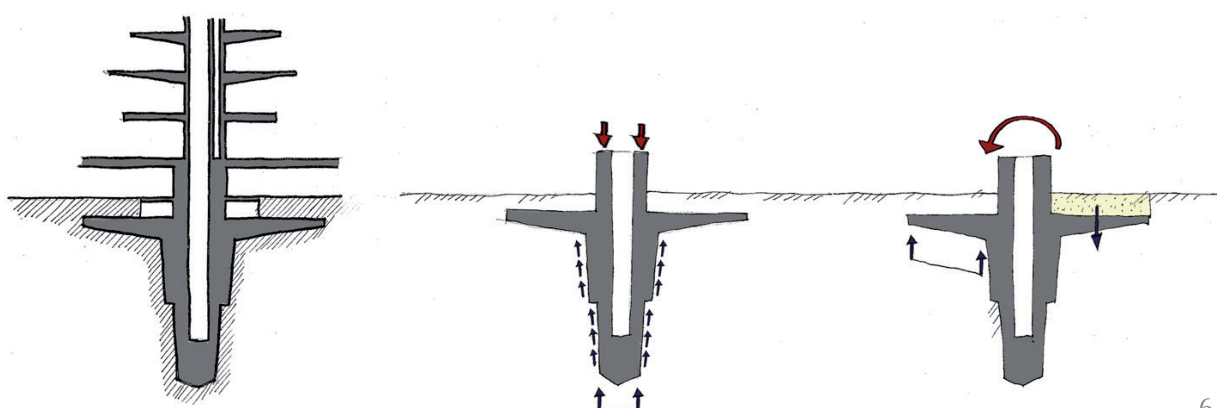


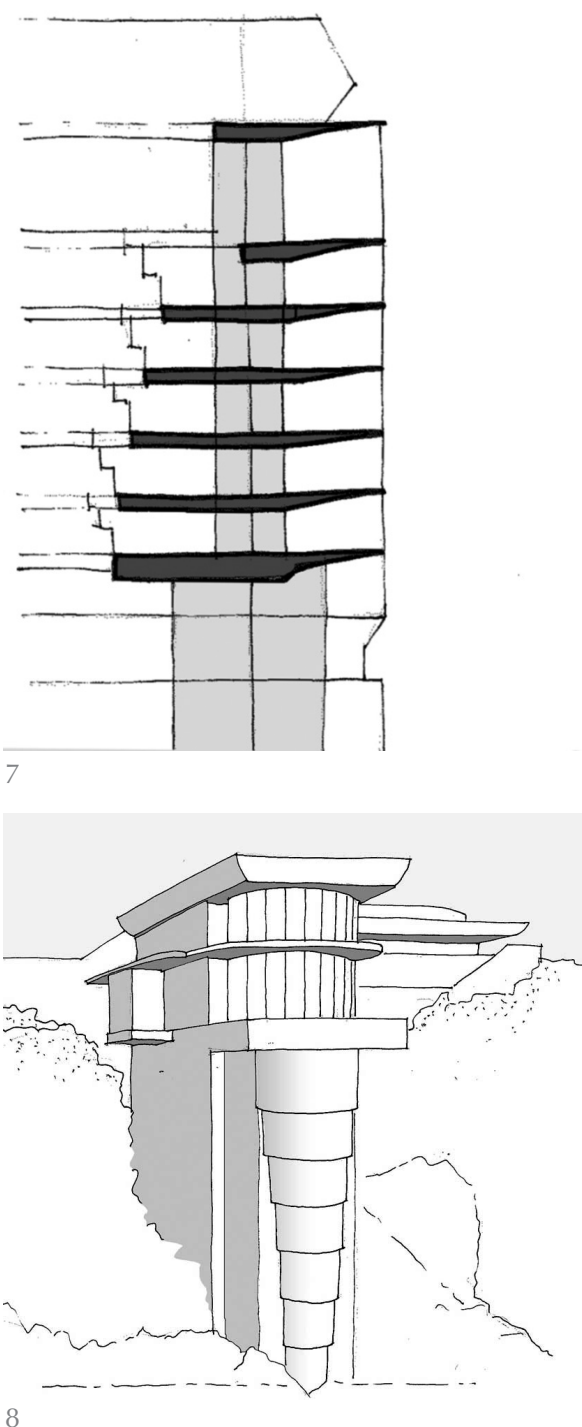

Las primeras propuestas que los ingenieros hicieron para la estructura del Guggenheim, hacia 1943, no satisfacían a Wright (17). Pensaba que colocaban demasiados pilares y eso iba en contra de la concepción de continuidad espacial del edificio. Polivka corroboró esa misma impresión, por lo que Wright le propuso resolver el problema mediante una lámina continua de hormigón, consiguiendo la estabilidad del conjunto sin apoyos interiores. Para ello era necesario utilizar complejos sistemas matemáticos así como el análisis fotoelástico del comportamiento de las uniones en los pilares. Estos puntos de la estructura son especialmente delicados a la hora de analizar el conjunto, ya que la variación de tensiones es muy acusada. Lo que verdaderamente les unió fue que ambos creían que la auténtica belleza proviene de la honradez estructural y de la naturalidad del comportamiento de los materiales, denominador común de la arquitectura orgánica. La predilección mutua por este aspecto convergió en la figura de Eduardo Torroja, con quien compartieron el interés hacia la lógica estructural de los materiales. Torroja estuvo en Taliesin con Wright y Polivka en 1950, y a partir de ese encuentro se fraguaron las bases del texto Razón y Ser de los Tipos estructurales (18), cuya primera edición se pretendía que fuese en Estados Unidos bajo el título Philosophy of Structures.

En abril de 1949 Polivka dio a conocer a Wright las obras de hormigón de Torroja, entre las que estaban el frontón de Recoletos o el puente sobre el Esla. El trabajo de Torroja impresionó gratamente a Wright, tanto que le generó interés por conocerle. De este modo, Polivka, organizó un viaje de Torroja a Estados Unidos. El encuentro se realizó en Taliesin en abril de 1950 y en la reunión estuvieron presentes Wright, Polivka y Torroja junto a dos miembros del Instituto (el ingeniero Nadal y el arquitecto Lucini), así como el editor de la revista Architectural Forum y el presidente de Corbetta Constructions, principal empresa constructora de Estados Unidos especializada en hormigón armado. De vuelta a España, Torroja escribió a Polivka: «...el principal recuerdo que ha quedado en mi mente es el del delicioso tiempo que pasé en San Francisco, así como las múltiples atenciones que ha tenido conmigo $»^{2}$.
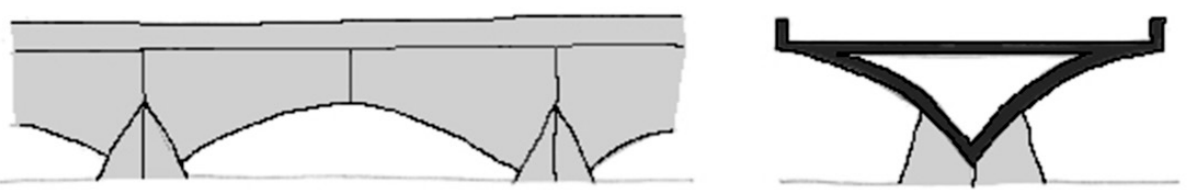

\section{Sección estructura \\ 8. Casa Morris. \\ 9. Alzado y sección puente Butterfly. \\ 10. Pabellón Belmont. \\ 11. Torre de la Milla.}

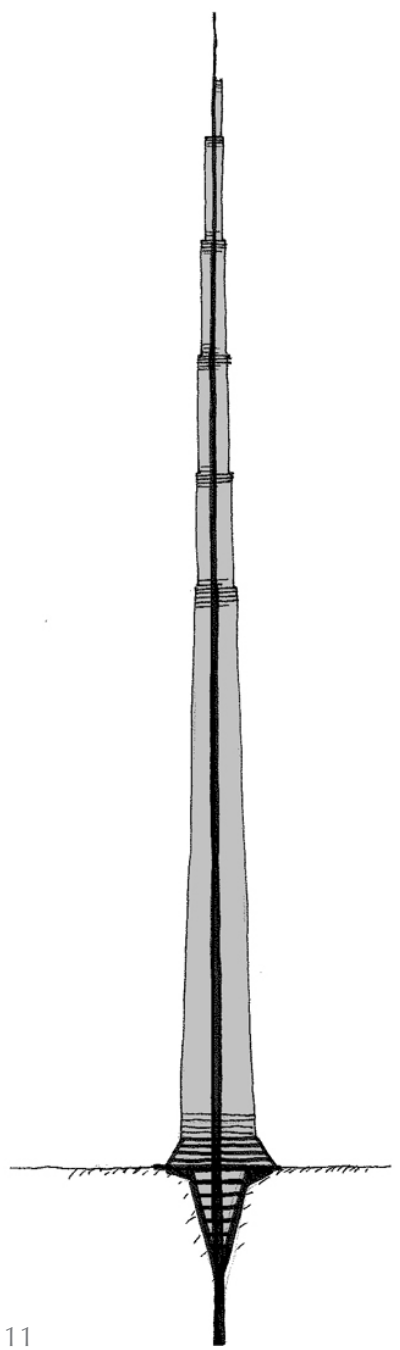

11

2 E.Torroja a J.J. Polivka. 31 de mayo de 1950. Polivka Papers, Folder 1.07

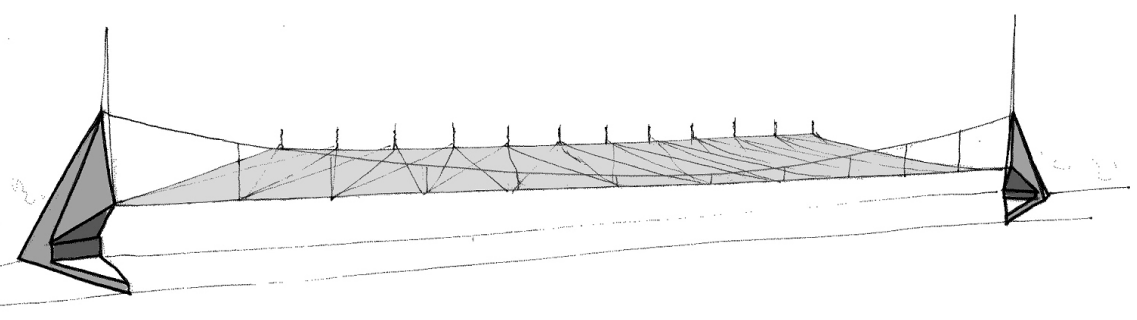


Parece ser que de las conversaciones entre Polivka y Torroja durante su estancia en Estados Unidos y de la correspondencia posterior entre ambos surgió la idea del texto Razón y Ser. En ese sentido Torroja le escribía: «Mi distinguido amigo: gracias por el interés que se ha tomado en la edición de mi libro, el título del cual bien podría ser The Philosophy of Structural Design. La idea del libro es llamar la atención de ingenieros y arquitectos sobre la importancia de elegir cuidadosamente el tipo de estructura a adoptar antes de tomar la decisión del diseño particular y, también, comentar varios aspectos del tema de manera fácilmente inteligible. No tengo ningún deseo de ir a argumentos matemáticos ni a problemas de cálculo, pero trato de hacer que el lector aprecie intuitivamente el fenómeno tensional y el problema de los esfuerzos en edificios como un aspecto integral de diseño. También intento indicar cómo la naturaleza de los materiales influencia el tipo de estructura, el método de construcción, etc... He redactado ya la mitad del libro, pero estoy demasiado ocupado y no sé cuándo podré acabarlo...»³.

Esta carta pone de manifiesto el interés de Polivka por la obra del ingeniero español. La idea de integrar en el diseño del edificio el comportamiento de su estructura y sus materiales era común tanto a Torroja como a Polivka y Wright. El edificio debía reconocer el carácter y planteamiento de su estructura y, a su vez, ésta debía servir a la arquitectura caracterizándola en la proporción que la misma requiriera.

Es destacable que Torroja sugiriese la redacción de un texto sobre estructuras sin el soporte de argumentos matemáticos, sino exclusivamente de carácter conceptual. Este aspecto evidencia la necesidad de valorar también este tipo de cuestiones, al margen del detalle de la resolución matemática del problema. En este sentido, Torroja enfatizó las cuestiones cualitativas de la estructura, aparte de la necesidad de cuantificar esfuerzos y tensiones, lo cual no se pone en duda.

Lo que Ilama la atención es que Polivka no haya sido reconocido igual que otros casos. Posiblemente su rol de ingeniero de estructuras quedó subordinado al del arquitecto, cuyo carácter, en este caso, enfatizó las diferencias. Durante los últimos años de su vida Polivka intentó recuperar un merecido reconocimiento, ofreciendo a varias revistas de arquitectura e ingeniería, como Architectural Forum o Consulting Engineer, aportar detalles respecto de su trabajo en colaboración con Wright. Este intento se materializó en varios artículos aunque, debido a la muerte de Polivka en 1960, no llegaron a tener demasiada difusión. En uno de esos textos Polivka escribió: "El Sr. Wright, con carácter excepcional, me pidió que pusiera mi nombre y posición en los dibujos y también en los informes y cálculos. Ciertamente estoy muy orgulloso de obtener el reconocimiento de este gran arquitecto. En su último libro -The Testament- me firmó con unas palabras halagadoras: Al buen doctor Polivka con estima y agradecimiento - Frank Lloyd Wright, $1958^{\prime \prime}$.

\section{LA PARTICIPACIÓN DE POLIVKA EN EL GUGGENHEIM MUSEUM}

La arquitectura de Wright tiene una componente estructural fuertemente intuitiva, por lo que desde sus primeras obras exploró las diferentes posibilidades tanto de los materiales y sistemas constructivos como de las tipologías estructurales (19). Cuando la envergadura de la estructura era menor, la intuición resultaba suficiente para la resolución del problema. Pero conforme la dimensión y el grado de integración de la estructura con el edificio fue adquiriendo importancia, Wright requirió la incorporación a su equipo de especialistas en esta materia, como Paul Mueller, antiguo colaborador de Adler y Sullivan para el edificio Larkin, y más tarde a Mendel Gleckman y William Wesley Peters, que desarrollaron estructuras tan singulares como las columnas dendriformes del edificio Johnson o los voladizos de la Casa de la Cascada (20). La inquietud de Wright por avanzar más terreno en este sentido estaba al límite de los conocimientos sobre hormigón de la época y en ese oportuno momento Polivka estableció contacto con él (21). Polivka proponía soluciones estructurales fuera del alcance de la mayor parte de ingenieros del momento, tanto respecto del hormigón como material estructural como de los métodos de análisis de sistemas complejos (22) (23).

La primera toma de contacto, a raíz del artículo de Wright en Architectural Forum, fue en febrero de 1946:

\section{«Estimado Sr. Wright:}

Le escribo como admirador suyo y de su trabajo desde hace tiempo, lo cual no significará mucho para Ud. porque estoy seguro que recibirá cartas similares a millares y probablemente esta carta pasará desapercibida.

Le admiro como ingeniero, a pesar de que, conforme a una cita suya en la última edición de Forum, "estos ingenieros sean unos completos malditos estúpidos". 
Puede que Ud. tenga razón porque los ingenieros, en sus concepciones estructurales, en muy raras ocasiones se guían por las eternas leyes de la naturaleza. Tome como ejemplo las telas de araña, que deberían ser estudiadas por los ingenieros cuya especialidad sea la construcción de puentes colgantes y redes estructurales bi- o tridimensionales.

El ingeniero medio sólo sabe de losas, vigas, vigas metálicas, columnas, etc. y cualquier desviación de esas herramientas cotidianas se considera algo inusual, loco o peligroso.

Durante muchos años he estado luchando contra este prejuicio. Su trabajo confirma y fortalece mis ideas y por ello le estoy muy agradecido.

Muy atentamente,

\section{J. J. Polivka» ${ }^{5}$.}

Wright le contestó breve, pero cordialmente: «Estimado Dr. Polivka: ¿Por qué no se acerca por aquí a vernos? Estaremos aquí hasta finales de mayo y Ud. será bienvenido en cualquier momento. Me gustaría hablar con Ud. Atentamente, Frank Lloyd Wright».

Polivka acudió con su mujer a Taliesin en Arizona, incorporándose durante unos días al taller. A Wright le gustaba que sus colaboradores se integrasen en las actividades de Taliesin, conviviendo durante las épocas de trabajo. Polivka quedó gratamente sorprendido por el buen ambiente que encontró, volviendo a su estudio de Berkeley con el encargo de estudiar y analizar las posibilidades de la estructura para el edificio del museo. Uno de los aspectos fundamentales que preocupaban a Polivka, al margen del análisis, era la puesta en obra de la estructura. Para ello, se puso en contacto con la empresa que consideraba de referencia, con experiencia en la construcción de grandes estructuras de hormigón armado y pretensado. La compañía Corbetta Construction Company mostró una buena disposición exponiendo su visión desde el punto de vista estrictamente constructivo.

Polivka iba transmitiendo a Wright rápidamente cualquier avance de la investigación, tanto sobre las cuestiones analíticas como en las gestiones que realizaba, con tal de afianzar la solución en voladizo tan controvertida. En las primeras aproximaciones incluyó un predimensionado de la losa con el fin de conseguir la rigidez necesaria del conjunto, sin penalizar demasiado el peso propio de la estructura «...una lámina de seis pulgadas $(15,24 \mathrm{~cm})$ asumiría todas los esfuerzos (momentos radial y tangencial, momentos de torsión y cortantes)...» $»^{6}$ Sin embargo, una losa de espesor tan reducido es extremadamente flexible $y$, aunque las deformaciones considerables no afecten a la seguridad de la estructura, pueden crear vibraciones alarmantes.

Ante la complejidad del problema, Polivka consideró necesario avanzar la investigación en paralelo de varias formas diferentes. Por un lado contando con la colaboración de empresas constructoras de cierta reputación y experiencia en la construcción de superficies singulares de hormigón armado. Por otro lado, afrontando el problema desde el punto de vista teórico, a través del planteamiento de las ecuaciones propias para el análisis de láminas (24).

Placa circular anular (Figura 12)

$$
\frac{d}{d r}\left[\frac{1}{r} \frac{d}{d r}\left(r \frac{d w}{d r}\right)\right]=\frac{V(r)}{D}
$$

\section{Donde:}

$w(r)$ : Deformación vertical (eje z) de un punto $r$ del plano respecto de la posición inicial.

$r$ : Distancia desde el centro a cualquier punto.

$V(r)$ : Esfuerzo cortante de un punto $r$ dentro de la placa.

$D$ : Rigidez de la placa, igual que en el caso de placas rectangulares

$$
D=\frac{E h^{3}}{12\left(1-\mu^{2}\right)}
$$

\section{E: Módulo de elasticidad del material}

$h$ : espesor de la placa

$\mu$ : coeficiente de Poisson

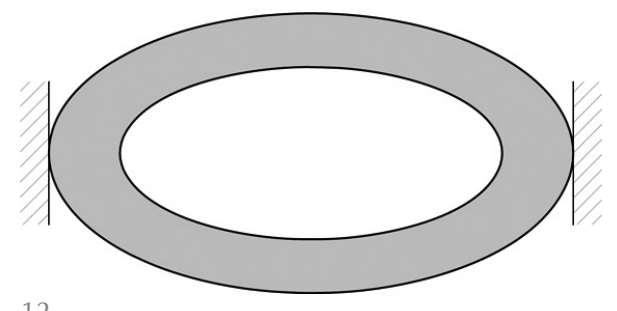

12

Cualquiera que se haya planteado la resolución de esta ecuación comprenderá cuán complicado resulta, especialmente en el caso de aplicar las condiciones contorno de la situación con el perímetro empotrado. Además, la complejidad geométrica de la estructura suponía realizar grandes simplificaciones del problema original, de manera que el análisis exclusivamente teórico resultaba ciertamente arriesgado, al no haber precedentes geométricos al respecto.

\section{Disco empotrado.}

${ }^{5}$ Polivka a Wright. 15 de febrero de 1946. Polivka Papers, Folder 1.02_01

${ }^{6}$ Polivka a Wright. 5 de junio de 1946. Polivka Papers, Folder 1.2_68.

7 TIMOSHENKO, S. - WOINOWSKI - KRIEGER, S. Theory of Plates and Shells. Ed. McGraw-Hill Book Company. Pág. 58 
13. Deformaciones de un disco plano $^{8}$.

14. Deformaciones de un disco abierto, menores que las del disco plano, por lo que el método elegido por Polivka se decanta

15, 16 y 17. Imágenes de una de las maquetas realizadas por Polivka como verificación del método de cálculo aplicado. Es significativo ver que se trata de un solo paso de la espiral, apoyado en el perímetro exterior. Este esquema corresponde con uno de los casos de Timoshenko, expuesto a continuación, y adoptados por Polivka para abordar el problema. ${ }^{10}$ por el lado de la seguridad.

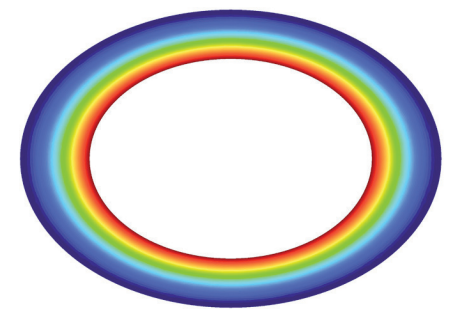

0
-0.0019673
-0.003935 $-0.0039345$ $-0.00078691$ $-0.0098363$ $-0.011804$ $-0.01377$ $-0.015738$

13

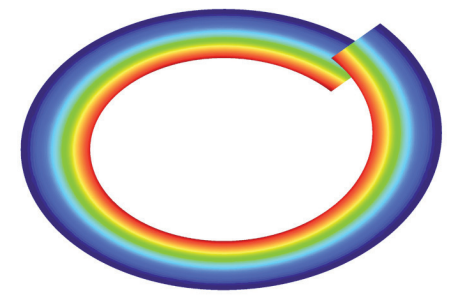

$-0.0013991$ $-0.0027981$ $-0.0041972$ $-0.00559654$ -0.0083944
-0.0097935 $-0.011193$

14

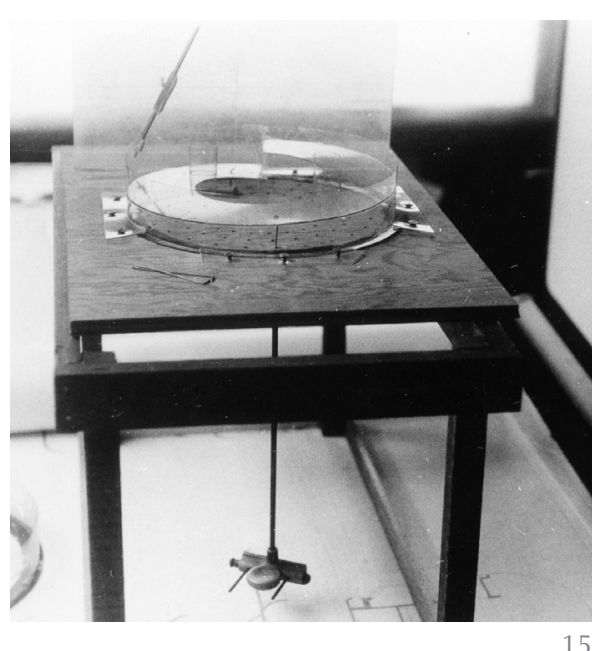

15

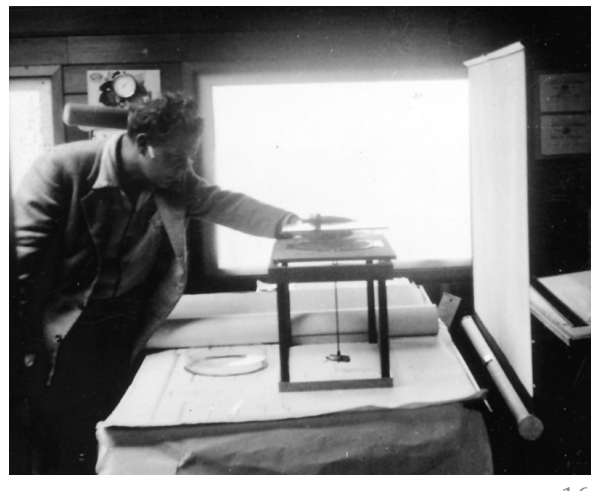

16

${ }^{8}$ Para los modelos se ha considerado una losa de hormigón de $15 \mathrm{~cm}$ de espesor, con un diámetro exterior de $26,6 \mathrm{~m}$ y uno interior de $17,8 \mathrm{~m}$, correspondiente a una planta intermedia del museo, bajo la carga de su peso propio.

${ }^{9}$ Polivka a Wright. 5 de junio de 1946. Polivka Papers, Folder 1.2_68.

10 Polivka Papers, fotos 3318, 3319 y 3326

${ }^{11}$ Polivka a Wright. 26 de Julio de 1946. Polivka Papers, Folder 1.02_79
La situación real suponía, no un disco cerrado empotrado en el perímetro (Figura 13), sino un disco abierto (Figura 14).

Por este motivo, Polivka consideró necesario complementar el análisis mediante la verificación de las deformaciones tomadas en maquetas realizadas en el laboratorio de la Universidad de Berkeley (Figuras 15, 16, 17). Las maquetas por sí solas no aportaban la precisión requerida, pero sirvieron para establecer un criterio que permitiese confirmar el acierto del planteamiento numérico. "...después de algunas reflexiones decidí abordar el problema primero teóricamente y posteriormente verificar los resultados obtenidos con ensayos en maquetas. $\mathrm{Ni}$ los ensayos ni el análisis por sí solos proporcionarían bases fiables para el diseño. ...Estamos preparando las maquetas para el ensayo y las pruebas mostrarán en qué medida el análisis teórico puede aplicarse a la estructura sometida a estados de carga más complejos y bajo la consideración de las dimensiones reales". ${ }^{9}$

Con estas maquetas Polivka pretendía poner a prueba la validez del método de análisis y clarificar una de sus principales preocupaciones; parametrizar el problema de manera que no tuviese que comenzar un nuevo análisis cada vez que fuese necesario variar la geometría de la rampa debido a cuestiones formales o programáticas. El funcionamiento estructural del edificio podía dividirse en cada uno de los niveles de la rampa, en cuyos casos las anchuras de la rampa y los diámetros interiores y exteriores variaban entre ellas y se fueron modificando también a lo largo del proceso. Por este motivo, y con tal de facilitar futuras adaptaciones, Polivka estableció un criterio general de dimensionado de la rampa en voladizo a partir de unos parámetros básicos. «...le envío hoy mismo la propuesta para simplificar el método de análisis del la rampa espiral alabeada, el cual puede aplicarse a las diferentes variaciones de anchura y diámetro exterior. Este método de análisis tiene un rendimiento de obtención de resultados muy similar al de los ensayos de maquetas» ${ }^{11}$.

El método parametrizable evaluado por Polivka había sido expuesto por Timoshenko en su libro sobre láminas. Como se ha puesto de manifiesto, el problema planteado para un disco empotrado bajo cargas repartidas resulta engorroso de operar, para el cual Timoshenko remite a una tabla (Figura 18) donde se dan coeficientes para el cálculo diferentes situaciones de carga y apoyos. 


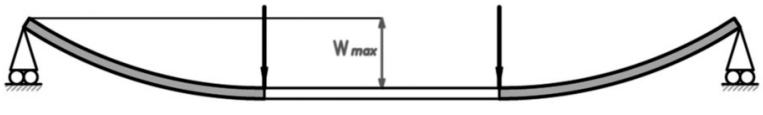

Caso 1

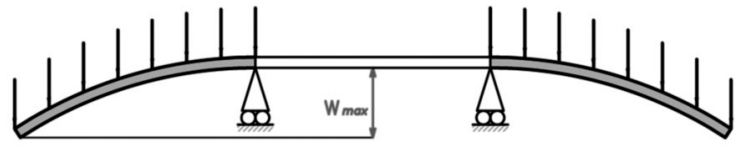

Caso 2

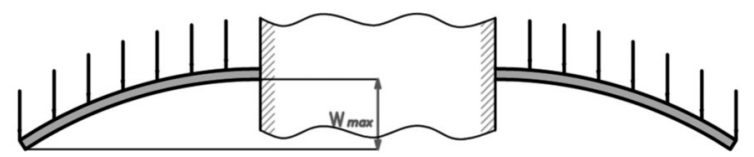

Caso 3
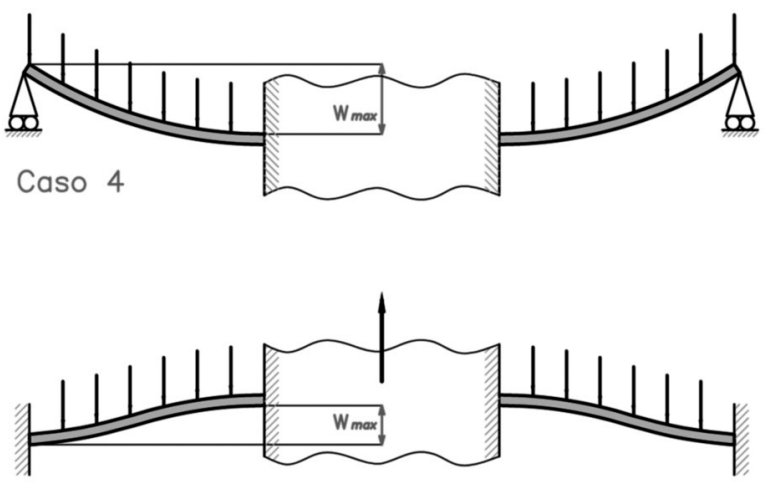

Caso 5

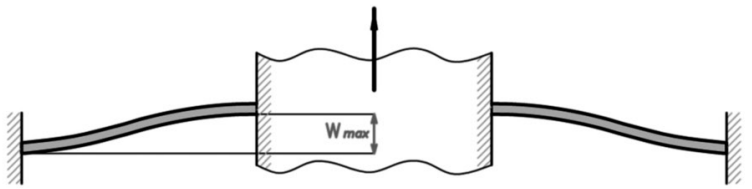

Caso 6

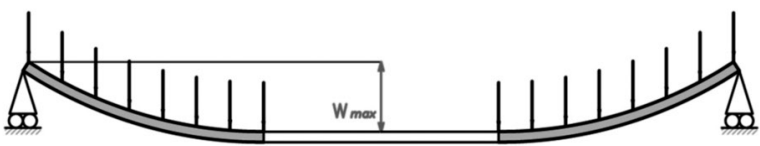

Caso 7

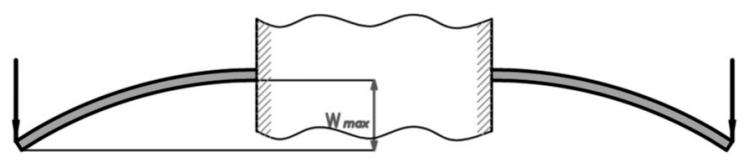

Caso 8

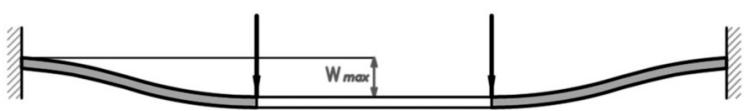

Caso 9

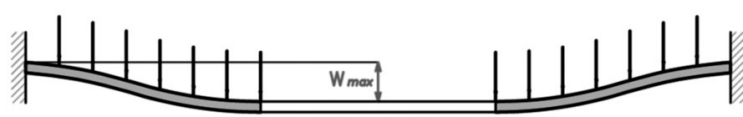

Caso 10
Esta tabla resume diez posibles situaciones habituales. Una vez obtenidas $k$ y $k_{1}$, se substituyen los valores en las siguientes expresiones $^{12}$ :

$$
\sigma_{\max }=k \frac{q a^{2}}{h^{2}}
$$

[6]

$$
\sigma_{\max }=k \frac{P}{h^{2}}
$$

$$
W_{\max }=k_{1} \frac{q a^{4}}{E h^{3}}
$$

Donde:

$\sigma_{\max }:$ Tensión normal máxima de la lámina tanto para una carga repartida $q$ como para una carga puntual $P$ aplicada en el extremo.
$W_{\max }:$ Deformación máxima de la lámina.

a: Radio exterior

$b$ : Radio interior

$h$ : Espesor de la placa

E: Módulo de elasticidad del material.

Mediante el método de superposición, Polivka pudo haber aplicado los coeficientes, corregidos para la $\mu=0,2$ del hormigón, de los casos 9 y 10 que correspondían con la situación de una de las plantas tipo. Es interesante señalar que los casos 3 y 8 , coinciden con la torre de investigaciones del edificio Johnson, que Polivka desarrollaba en paralelo al proyecto del museo.

La evolución de la propuesta inicial de Wright seguía su curso y Polivka no sólo se limitaba a predimensionar los espesores de las láminas de hormigón o a verificar estrictamente su funcionamiento, sino que
18. Relación de los casos expuestos en la teoría de placas de Timoshenko. ${ }^{13}$

12 TIMOSHENKO, Stephen P. - WOINOWSKY-KRIEGER, S. Teoría de placas y láminas. Espasa-Calpe, Madrid 1957. Tabla 3, págs. 80 y 82.

${ }^{13}$ TIMOSHENKO, Stephen P. - WOINOWSKY-KRIEGER, S. Teoría de placas y láminas. Espasa-Calpe, Madrid 1957. Figura 36, pág. 81. 
también sugería la introducción de modificaciones en la estructura que repercutían en el resultado arquitectónico, como la geometría de la propia rampa, la posible función estructural de la barandilla y la fachada o la importancia de las dos columnas frente al núcleo anteriormente comentadas. Todo el trabajo de Polivka giraba en torno al concepto espacial de continuidad planteado por Wright, basado en el planteamiento estructural del voladizo de la rampa hacia el interior.

«Los resultados presentados en los modelos demuestran que su concepción de la rampa alabeada en espiral con el borde interior cóncavo es segura (como yo había previsto y así manifesté a su yerno Wes Peters), y se demuestra por lo siguiente:

1. La rampa alabeada en espiral tiene substancialmente mayor rigidez $y$, su máxima deformación se reduce un $40 \%$ aproximadamente. La curvatura tangencial de la rampa proporciona a la placa el valor característico de una lámina, reduciendo así la intensidad de los momentos flectores y consecuentemente las deformaciones.

2. El borde interior cóncavo de la rampa es el otro factor que aumenta la rigidez y la capacidad portante de la losa.

3. Los apoyos creados por las dos columnas circulares huecas funcionan como refuerzo añadido de la rampa, especialmente en la proximidad de dichas columnas» ${ }^{14}$

A diferencia de las prácticas habituales actuales, donde todo está regulado códigos normativos, en el momento de la redacción del proyecto de la Modern Gallery ciertas cuestiones podían quedar sujetas al criterio del proyectista. En este caso, la aplicación de la sobrecarga de uso resultaba de especial importancia, ya que una cuantificación demasiado conservadora podría dificultar la situación hasta el punto de transformarla en inviable. También aspectos del análisis en detalle resultaban de crucial importancia. La mayor concentración de esfuerzos en la rampa se focaliza alrededor de la intersección con los soportes, donde el gradiente de esfuerzos es muy acusado y tanto un análisis numérico simplificado como la elaboración de maquetas generales podían pasar por alto aspectos cruciales para el conjunto. Esta cuestión Polivka planteaba abordarla a través de la elaboración de maquetas en detalle analizadas mediante foto-elasticidad. "Desde que le escribí enviándole el informe sobre el modelo de tensiones me he dado cuenta de que no he analizado la seguridad de los soportes de la rampa espiral. Este elemento estructural, en mi opinión, es la clave de todo el diseño y creo que podría añadir al informe cómo se puede resolver este problema... Estoy preparando una maqueta de detalle del encuentro para ensayarla mediante fotoelasticidad, ya que el método habitual de diseño no se puede aplicar en este tipo de elementos estructurales». ${ }^{15}$

Wright no dudaba de las bondades del análisis de Polivka e iba incorporando las sugerencias que éste le proponía a excepción de los soportes radiales en el exterior de la rampa. Así le escribía el 27 de junio y 11 de julio de 1946: «Mi querido Dr. Polivka: Hemos estado esperando las páginas del informe y éstas acaban de llegar. La tesis ha sido interesante y puede ser posible incorporar sus sugerencias. ${ }^{16}$...la cuestión que me ha enviado es muy interesante y probablemente muy válida para nosotros. Tan pronto como tenga tiempo me introduciré en el problema cuidadosamente, tendrá noticias mías directamente. Frank Lloyd Wright». ${ }^{17}$

Aunque las respuestas de Wright eran breves, Polivka sentía que su trabajo servía de gran ayuda en el desarrollo del proyecto del museo y esto hacía crecer su entusiasmo por el encargo. A mediados de agosto de 1946 Polivka había avanzado ya considerablemente en la investigación. Para Wright este trabajo resultó de gran utilidad, pero, Ilegado a este punto y ante la incertidumbre sobre la ejecución de las obras, no deseaba seguir costeando las horas de trabajo de Polivka. «...por ahora no quiero incurrir en más gastos de los que usted ya ha cargado en mi cuenta. Indudablemente lo que usted ha hecho (y hará) me será de gran ayuda cuando el nuevo Museo sea construido. Estamos esperando la oportunidad de construirlo». ${ }^{18}$ En ese momento, la situación económica mundial no era muy favorable, por lo que Polivka, a pesar de su especial interés por continuar su dedicación, también requería de las correspondientes compensaciones económicas, como es normal. Aún así, siguió trabajando y enviando información a Wright, acompañando sus comentarios particulares del análisis con elogios hacia el edificio y a la figura del arquitecto. A pesar de la situación, Polivka tenía especial empeño en concluir el estudio que confirmase la viabilidad de la solución sin soportes en el borde interior de la rampa. El informe iba también encaminado a la justificación de la estructura frente a las autoridades de New York, cuestión que resultaba necesaria en las situaciones en las que la estructura no se ajustaba a los 
patrones conocidos y regulados en la construcción habitual: entramados de acero formados por pilares y vigas.

A finales de 1946, y con la intención de aliviar el resultado económico de la construcción, en Taliesin exploraban otras posibilidades con tal de ajustar al máximo el coste de la estructura del edificio. Una de las vías estudiadas fue la de transformar la estructura en un entramado de acero, situación que Polivka no llegó a rechazar directamente, aunque, a la vista de sus pasos, no parecía ser demasiado partidario. De este modo, siguió con la investigación en el plano económico y constructivo, contando con la colaboración de la compañía Corbetta, ya mencionada anteriormente.

«Apreciado Sr. Corbetta: En referencia a su carta del 7 de mayo, olvidé informarle que la estructura mediante lámina de hormigón que le mencioné en mi carta anterior está en el último estado de diseño y me gustaría discutir el trabajo con usted próximamente. Hay un esquema alternativo en estudio mediante esqueleto de acero embebido en hormigón y una discusión en persona podría aclarar algunos problemas que he encontrado en esta inusual construcción. Estoy planificando ir a New York la segunda mitad de diciembre. Por favor, hágame saber si estaría disponible para hablar de este asunto. La cuestión principal es elegir la estructura más económica bajo las condiciones actuales». ${ }^{19}$

Frente a esta actitud perseverante de Polivka y viendo que el coste de la investigación de la estructura de la Modern Gallery se incrementaba, Wright se dirigió a Polivka de forma contundente, para que interrumpiese su trabajo.

Posiblemente Polivka dejó de pasar más facturas a Wright, pero no canceló las entrevistas que tenía concertadas para ese mes de diciembre en New York con Mr. Corbetta, con la intención de tratar diferentes aspectos de la ejecución de las láminas de hormigón, y con el Departamento de Construcción de New York. Polivka continuó informando a Wright puntualmente sobre las consultas realizadas $y$, a pesar de que intentaba no poner de manifiesto el conflicto entre un entramado de acero y la solución de láminas de hormigón, sus pasos iban claramente encaminados hacia esta última solución. «...Preparando los dibujos finales para la Modern Gallery en New York, debemos tener resueltos los últimos detalles típicos. No importa si el sistema de la rampa es de hormigón armado o mediante un entramado de acero embebido en el hormigón, será necesario disponer refuerzos postensados con el fin de prevenir fisuras e incrementar la resistencia de la estructura. Estoy pensando en un nuevo tipo de refuerzo pretensado que ha desarrollado muy recientemente el Dr. F. von Emperger. Yo estuve asociado con él en Viena hace bastantes años. Su método de pretensado tiene muchas ventajas, como el ahorro de una considerable cantidad de acero de alta calidad, mayor uniformidad y facilidad de pretensado, relativamente pequeña cantidad de refuerzos típicos adicionales y una menor fisuración del hormigón; en definitiva, cuestiones para garantizar una mayor durabilidad de la estructura». ${ }^{20}$

Este es el momento en el que parece que finalizó la primera etapa de colaboración entre Wright y Polivka. El proyecto se encontraba bloqueado por motivos económicos y, por parte de Wright, una de las posibles soluciones al respecto consistía en transformar la estructura en un entramado de acero. Polivka no veía argumentos técnicos objetivos para rechazar esta opción, pero intuía también cuáles eran las virtudes de la solución de hormigón desde el punto de vista arquitectónico. Por este motivo buscó la manera de resolver los dos aspectos especialmente conflictivos del planteamiento estructural: el económico y la aceptación por parte de las autoridades de New York de la intrépida solución de lámina de hormigón. Hay que señalar que el presupuesto total no debía sobrepasar los 1,5M\$ y sólo la estructura de hormigón suponía más de la mitad de esta cantidad. Wright, además, debía sortear las dificultades derivadas de planteamientos más generales, como la disposición de los cuadros a lo largo de la rampa, las particularidades del programa o las constantes ampliaciones del solar.

Polivka siguió desarrollando el mismo tipo de trabajo que hasta el momento $y$, finalmente, durante 1952 la estructura quedó definida. Su labor en esa fase se centró especialmente en las justificaciones frente a la comisión de la construcción de la ciudad de New York, ya que la tipología estructural no era muy habitual en la construcción de edificios en ese momento. A pesar de las dificultades, las incertidumbres sobre la ejecución y utilización del sistema novedoso se fueron resolviendo hasta el comienzo de la construcción en 1956.

\section{APORTACIONES DE POLIVKA A LA ARQUITECTURA DE WRIGHT}

Es evidente que el Guggenheim tal y como lo conocemos ahora es deudor de las aportaciones de Polivka, no sólo en el sentido
19 Polivka a Corbetta. 24 de noviembre de 1946. Polivka Papers, Folder 1.03_80.

${ }^{20}$ Polivka a Wright. 19 de enero de 1947. Polivka Papers, Folder 1.04_01. 
práctico de haber resuelto los pormenores técnicos de la estructura, sino fundamentalmente por el planteamiento de la misma y lo que ésta representa en el conjunto del edificio. En la arquitectura de Wrigh, así como es difícil separar la estructura del global del edificio, las aportaciones realizadas por Polivka también se difuminan en cierto modo dentro de todo el proceso de trabajo. Es importante hacer hincapié en el carácter fuertemente dominante de Wright, que en ocasiones pudo eclipsar a sus colaboradores. Polivka era consciente de este hecho y durante los últimos años de su vida reivindicó sus aportaciones, aunque sin demasiado éxito, frente a la crítica arquitectónica del momento.

Desde el primer contacto entre ambos a propósito del museo, Polivka se desmarcó de la práctica habitual de los ingenieros de estructuras, basada en la aplicación de sistemas de entramados de pilares y vigas, siendo conocedor de las posibilidades laminares del hormigón. Esa visión diferente de la estructura es lo que despertó en Wright el interés por trabajar con él. El proyecto del museo se basaba en un planteamiento estructural de continuidad espacial donde los sistemas de entramado, más tradicionales, no se ajustaban a los requerimientos formales de Wright. Polivka era conocedor de la naturaleza del hormigón armado y pretensado como material con posibilidades mucho más amplias que las estrictamente necesarias para formar retículas. Wright había ido demostrando durante diferentes obras, desde la construcción del Templo Unitario en 1910, su interés por el material y su capacidad por concretar sus aspiraciones arquitectónicas. Pero el proyecto del museo tal como se planteaba en 1946 excedía la capacidad de la mayor parte de los ingenieros de estructuras para abordar un problema de esas dimensiones.

En una de sus primeras respuestas sobre la estructura, Polivka propuso una lámina de seis pulgadas de espesor para resolver la rampa, a falta de una verificación de este espesor mediante la realización de los ensayos con maquetas. Esta fue una visión directa del resultado final que, al margen de las particularidades de las versiones intermedias, se mantuvo hasta la construcción y permitió que la imagen sin soportes interiores que Wright deseaba se pudiese llevar a cabo.

La mayor parte de las aportaciones que Polivka realizó a la arquitectura de Wright se concentran en soluciones con estructura de hormigón y novedosos métodos para su análisis (25)(26). La torre de investigaciones del edificio Johnson es, junto con el Guggenheim, el otro de los dos únicos ejemplos construidos. La estructura de la torre forma parte de la tipología de edificios en altura, pero la concepción de la misma estaba íntimamente ligada a la del museo, a través del voladizo. En las otras colaboraciones, especialmente en el Rogers Lacy Hotel y la casa Morris, el tema del voladizo resultó también ser fundamental y se resolvió mediante una estructura de hormigón fuera de los límites convencionales. En el puente Butterfly, aunque no se trataba de una estructura propiamente en voladizo (por lo menos en sentido longitudinal, no así en el transversal), sin embargo se basó en la aplicación de las características del hormigón para la construcción de puentes, donde Polivka tenía una amplia experiencia. Los casos del Belmont Racetrack Pavilion o de la torre de la Milla implicaron la utilización de sistemas atirantados de cables, más allá de los límites propios del hormigón.

La cuestión es que Wright requería de las herramientas para que sus intenciones pudiesen materializarse en las propuestas de mayor envergadura $y$, en ese sentido, Polivka resultó ser el apoyo necesario, principalmente al potenciar los valores de su arquitectura a través de la estructura y sus materiales.

\section{CONCLUSIÓN}

A través de la extensa correspondencia entre Wright y Polivka se demuestra que hubo una estrecha colaboración entre ambos. La contribución del ingeniero a la obra de Wright merece una consideración especial, satisfacción que Polivka persiguió hasta su muerte sin demasiado éxito. En su etapa europea había demostrado su talento abordando la resolución de problemas fuertemente vinculados a los aspectos formales de la estructura, pero, respecto de su etapa de colaboración con Wright, éste nunca hizo reconocimiento público de sus colaboradores, e incluso en trabajos como el de Butterfly Wings Bridge, atribuible en gran parte a la contribución de Polivka, Wright se preocupó de promocionarlo como propio. Esta situación, salvo en raras ocasiones, ha sido habitual en la arquitectura actual, dejando en un segundo plano las aportaciones de muchos ingenieros de estructuras. No obstante, Wright, de algún modo, manifestó su gratitud hacia de Polivka incluyéndolo en una lista de los ingenieros de estructuras más influyentes, como Roebling, Maillart, Torroja o Nervi, que plasmó en uno de los dibujos de la Torre de la Milla. 
En cuanto a la estructura del museo, queda de manifiesto cuales fueron los métodos de análisis que aplicó Polivka, aplicando un gran esfuerzo de abstracción coherente con los planteamientos arquitectónicos de Wright. El funcionamiento de la estructura, aparentemente complejo, se puede resumir como una superposición de discos abiertos empotrados en su perímetro, dejando como contribuciones secundarias la rigidez de barandillas y fachadas de hormigón. Está claro que este esfuerzo no hubiese sido posible sin los conocimientos específicos de Polivka, ni sin su visión integral de la estructura con la arquitectura (Figura 19).

\section{AGRADECIMIENTOS}

El autor quiere mostrar su agradecimiento al archivo de la Universidad de Buffalo, de donde proceden las imágenes y textos ${ }^{21}$ referentes a Polivka, a José Angel Sanz, doctor arquitecto, por sus recomendaciones a la hora de indagar en la obra de Frank Lloyd Wright a través de la amplísima bibliografía existente al respecto, y a Robert Brufau, doctor arquitecto, por haber tenido la paciencia de dirigir mi tesis doctoral y sobre todo haber orientado mis pasos profesionales hacia la comprensión de la estructura como una componente fundamental de la arquitectura.

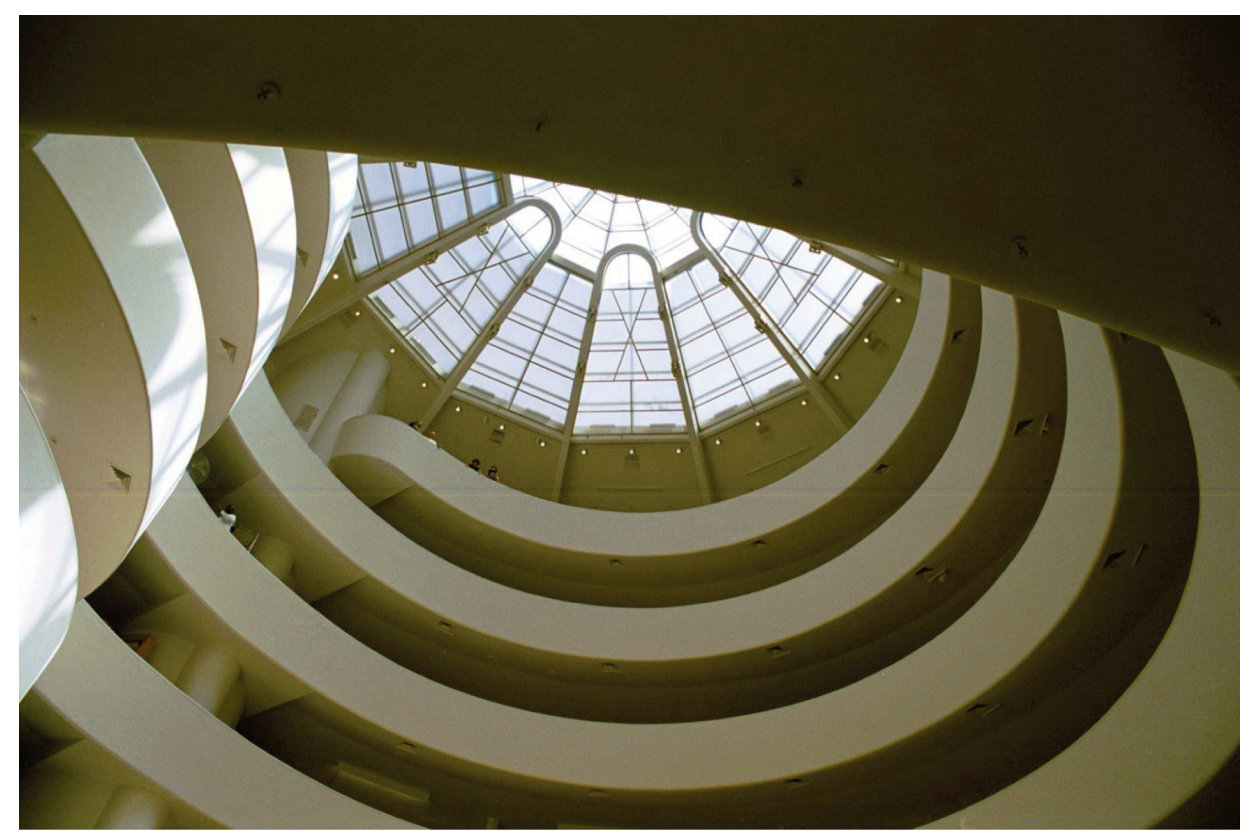

19

\section{REFERENCIAS}

(1) Martín-Sáiz, Diego. (2012 ). El Guggenheim Museum de New York. Interpretación del papel de la estructura a través de la colaboración entre Frank Lloyd Wright y Jaroslav J. Polivka. Universitat Politècnica de Catalunya, enero.

(2) Margolius, I. (2002). Architects + Engineers = Structures. Wiley-Academy. London.

(3) Churtichaga, J. M. (2008 ). La estructura veloz. Revista Consejo Superior Colegios Arquitectos de España, Marzo.

(4) Fernandez Troyano, L. (2005). Arquitectos e ingenieros, historia de una relación. Revista de Obras Públicas, 3460: 45-54, Noviembre.

(5) Fornies, L. S. (1983). Del arquitecto filósofo al ingeniero constructor. Un debate sobre el Arte y la Ciencia en el Siglo XIX. Revista de Obras Públicas, 3210: 29-31.

(6) Komentdant, A. E. (2000). 18 años con el arquitecto Louis I. Kahn. Colegio Oficial de Arquitectos de Galicia.

(7) Aguiló, M. (2008). Estructuras para los edificios singulares españoles. ACS, Madrid.

(8) Azagra, D., Bernabeu, A. J. (2012). La estructura de las formas libres. Informes de la Construcción, 64(526): 133-142, doi: 10.3989/ic.2012.v64.i526.

(9) Manterola, J. (1998). La estructura resistente en la arquitectura actual (continuación). Informes de la Construcción, 50(456-457): 15-45, doi:10.3989/ic.1998.v50.i456-457.

(10) Manterola, J. (2005). La estructura resistente en la arquitectura actual. Informes de la Construcción, 57(499-500): 9-35, doi:10.3989/ic.2005.v57.i499-500.

(11) Mainstone, R. J. (1983). Developments in structural form. Penguin Books.

19. El sistema de soporte de la rampa, acorde con la concepción espacial de continuidad proyectada por Wright

\begin{abstract}
${ }^{21}$ Las imágenes correspondientes al retrato de Polivka y las maquetas realizadas para los ensayos proceden del Archivo de la Universidad de Buffalo, New York. También proceden del mismo archivo la correspondencia mantenida entre Wright y Polivka desde 1946 hasta 1959 así como diversos artículos y textos escritos por Polivka, la mayor parte de ellos no publicados.
\end{abstract}

El resto de imágenes, las ilustraciones de los proyectos de Wright en colaboración con Polivka y los esquemas explicativos, han sido elaborados por el autor. 
(12) Siegel, C. (1966). Formas Estructurales en la Arquitectura Moderna. Compañía Editorial Continental. México.

(13) Zannos, A. (1987). Form and structure in architecture. The role of statical function. Van Nostrand Reinhold. New York.

(14) Frampton, K. (1994). Modernization and mediation: Frank Lloyd Wright and the impact of technology. Artículo del catálogo de la exposición sobre Wright en el MOMA en 1994: Frank Lloyd Wright, Architect.

(15) Muskat, B. A. (2000). Engineering the Organic, an investigation into the Collaboration of Jaroslav Joseph Polivka and Frank Lloyd Wright. Tesis doctoral Universidad del Estado de New York en Buffalo.

(16) Quinan, J. (1988). L'ingegneria e gli ingegneri di frank Lloyd Wright. Casabella, 52: 42-53.

(17) VV.AA. (1946). Architectural Forum. Frank Lloyd Wright. Enero.

(18) Torroja, E. (1957). Razón y ser de los tipos estructurales. Instituto Eduardo Torroja. Madrid.

(19) Levine, N. (1996). The Architecture of Frank Lloyd Wright. Princeton Univertity Press, New Jersey.

(20) Scully, V. Frank Lloyd Wright. Ed. Bruguera, Barcelona 1961.

(21) Castro Chicot, J. R. (2000). Frank Lloyd Wright y el Guggenheim Museum. Tesis doctoral. ETSAB. Universidad Politécnica de Cataluña. Barcelona.

(22) Dal Co, F. (2004). Il tempo e l'architetto. Frank Lloyd Wright e il Guggenheim Museum. Ed. Electa, Milano.

(23) Trombetti, T. (2007). Come è stato construito il Guggenheim Museum di Frank Lloyd Wright. Casabella, 760: 48-61.

(24) Timoshenko, S. P., Woinowsky-Krieger, S. (1957). Teoría de placas y láminas. EspasaCalpe. Madrid.

(25) Polivka, J. J. (1942). Analysis of Gravity Load Stresses by Photoelasticic Methods. Illinois Institute of Technology

(26) Polivka, J. J. (1946). Experimental Stress Analysis of Caisson Gates in the Kaiser Shipyards, Richmond, California. Society for Experimental Strees Analysis in Buffalo. 